\title{
Constraining Modified Gravity Models Using the Thermal Sunyaev- Zeldovich Effect
}

\section{Ivan De Martino*}

Department of Theoretical Physics and History of Science, University of the Basque Country UPV/EHU, Faculty of Science and Technology, Barrio Sarriena s/n, 48940 Leioa, Spain

\begin{abstract}
Some modified gravity theories add, in their weak field limit, a Yukawa-correction to the Newtonian gravitational potential. Such a correction usually depends on the two parameters, one that accounts for the modification of the gravitational constant, and another one representing the scale length on which the scalar field propagates. The thermal Sunyaev-Zeldovich temperature anisotropies can be used to test the modified gravitational potential well demonstrating that the Yukawa-like gravitational potential is able describe the distribution of the hot Intra Cluster Medium without accounting for a Dark Matter halo.
\end{abstract}

Keywords: Alternative theories of gravity; MOG; Galaxy cluster; Dark matter; Dark energy; Cosmic microwave background; SunyaevZeldovich effect

\section{Introduction}

Our knowledge about the Universe was increased through the centuries, and observations have quickly improved and became more accurate in the last three decades. We have entered the era of precision cosmology where the observables have been determined within a few percent accuracy [1-6]. The observations indicate that baryons only contribute a few percents to the total amount of the matter and energy in the Universe, and the latter is ongoing a period of accelerated expansion. These data are well accommodated within the framework of the concordance cosmological model (also named $\Lambda \mathrm{CDM}$ model). The model explains the evolution of the Universe from the first fractions of a second to the present day. To account for these observations, the concordance cosmological model that is entirely based on General Relativity (GR), assumes the existence of two extra energy-density components: i) the Dark Matter (DM), and ii) a cosmological constant $\Lambda$, equivalent to a perfect fluid with negative pressure, or its generalizations usually named Dark Energy (DE). The DM is characterized by a small temperature, it interacts only gravitationally with the other components, and it constitutes about a $26 \%$ of the total amount of energy-density of the Universe. It allow us to explain the emergence of the Large Scale Structure and the dynamics of self-gravitating systems in the framework of GR. The DE accounts for a $\sim 68 \%$ of the total energy-density, and it is required to explain the current period of accelerated expansion [6]. From one hand, the dynamical effects of both DM and DE on large scales are very well constrained; on the other hand, the lack of evidence of counterparts at the particle level can be interpreted as a breakdown of GR at scale beyond the Solar System. Thus, alternative models to GR have been proposed to explain both the dynamics of self-gravitating systems and the cosmological expansion history without resorting to extra components.

Broadly speaking, these alternative models, usually named Extended Theories of Gravity (ETGs), generalize the Hilbert-Einstein Lagrangian by including higher-order curvature invariants and minimally or nonminimally coupled terms between scalar fields and geometry. Such higher-order theories contain extra degrees of freedom that, in the weak field limit, can be recast as new gravitational scale lengths. The general paradigm for $(2 k+2)$-order theories of gravity demonstrates that, a new characteristic scale length arises in Newtonian limit increasing the theory of two derivation orders [7]. Thus, gravity is not longer scale invariant, and DM and DE could be interpreted as the effect of high order theories on scale larger than the Solar system one. This review is focused on two ETGs: (a)the well known $f(\mathrm{R})$-gravity that replaces the Ricci scalar, $R$, in the Hilbert-Einstein action with a more general function of the curvature $f(\mathrm{R})$ for comprehensive reviews see [8-12]; and (b) the more recent Scalar-Tensor-Vector Gravity theory (STVG), also known as MOdified Gravity (MOG), that adds scalar, tensor and massive vector fields to the standard Hilbert-Einstein action (for more details see $[13,14]$. Both ETGs introduce a Yukawa-like correction to the Newtonian gravitational potential in their weak field limit [13-16]. In $f$ (R)-gravity, such correction term is characterized by two parameters the strength $\delta$ and the scale length $L$ of the Yukawa-term that are related to the additional degree of freedom/scalar fields arising from the theory. In MOG theory, the mass of the vector field and its strength are governed by two running constants, $\alpha$ and $\mu$, that are promoted to scalar fields and can be constrained by data.

Both theories have been tested from the astrophysical to the cosmological scales. Specifically, $f(\mathrm{R})$-gravity is able to describe the star formation and evolution $[17,18]$, the emission of gravitational waves for binary systems $[19,20]$, the galactic rotation curves in spiral galaxy [21], the dispersion velocity in elliptical galaxy [22], the dynamics of gas in galaxy clusters [23-25], and the emergence and evolution of the Large Scale Structure [26]. On the other hand, MOG is able to describe the dynamics of self-gravitating system from galactic to extragalactic scales[27-30] and, at the same time, it can explain the evolution of the Universe as a whole [31-33]. Despite their successes, there is not definitive proof about the need of modifying gravity; nowadays, ETGs

*Corresponding author: Ivan De Martino, Department of Theoretical Physics and History of Science, University of the Basque Country UPV/EHU, Faculty of Science and Technology, Barrio Sarriena s/n, 48940 Leioa, Spain, Tel: +34 946 0120 00; E-mail: ivan.demartino1983@gmail.com

Received September 01, 2017; Accepted September 25, 2017; Published September 29, 2017

Citation: De Martino I (2017) Constraining Modified Gravity Models Using the Thermal Sunyaev-Zeldovich Effect. J Astrophys Aerospace Technol 5: 150. doi:10.4172/2329-6542.1000150

Copyright: () 2017 De Martino I. This is an open-access article distributed under the terms of the Creative Commons Attribution License, which permits unrestricted use, distribution, and reproduction in any medium, provided the original author and source are credited. 
just represent a valid alternative to the $\ddot{E}$ CDM model to overcame some of its shortcomings. Neverthless, having alternatives demand to test them in all possible physical scenarios.

\section{Literature Review}

Here we briefly review the chance offered by the thermal SunyaevZeldovich (TSZ, [34]) effect in galaxy clusters to constrain both $f(\mathrm{R})$ and MOG theories. We will show that the predicted effect agrees with the observed one when the intra cluster gas is in hydrostatic equilibrium within the modified Newtonian potential. There is no need, in these models, for introducing a dominant DM halo component. This review is organized as follows: in sect. II, we briefly describe the main features of galaxy clusters and the TSZ effect; in sect. III, we describe the methodology used to test ETGs $[24,25,30]$. Specifically, we illustrate the data, the model and the statistical analysis; in sect. IV, we highlight the results and, finally, in sect. $\mathrm{V}$ we give the conclusion.

\section{Cluster of Galaxies}

Galaxy clusters are the largest virialized objects in the Universe, with a virial mass in the range from $10^{13}$ to (few) $10^{15} \mathrm{M}$. The mass of baryons in cluster is composed at least by two components, diffuse Intra Cluster Medium (ICM), and stars. Neverthless, most of baryons are not in galaxies but they are in the diffuse ICM [35-37].

Clusters contain from hundreds to up to one thousand galaxies within $2 \mathrm{Mpc}$ from their center. They account for a a mere 3\% of the total mass of the cluster, while hot ICM gas contains most of the baryons up to $12 \%$ of the total mass. The remaining $85 \%$ is usually associated to a DM halo. The ICM is highly rarefied: electron number densities are typically $n_{e} \sim 10^{-4}-10^{-2} \mathrm{~cm}^{-3}$, but it has a temperature in the range from $10^{7}$ to $10^{8} \mathrm{~K}$, thus clusters are strong X-rays sources with a luminosity $L_{X} \sim 10^{43}-10^{45} \mathrm{erg} / \mathrm{s}$. The size of the cluster are typically given in term of the radius $r_{500}$, defined as the radius at which the mean overdensity of the cluster is 500 times the critical density of the Universe at the same redshift $\left(\rho_{c}(z)\right)$, and the corresponding enclosed mass is $M_{500} \equiv \frac{4 \pi}{3}\left[500 \rho_{c}(z)\right] r_{500}^{3}$. These definitions are rather convenient since scaling relations based on numerical simulations and $\mathrm{X}$-ray observations allow to determine $r_{500}$ for individual clusters $[38,39]$. Scaling relations can be also used to test the physics of clusters of galaxies[40-47].

The interest for obtaining a good description of the distribution of the ICM within the gravitational potential well generated by the DM distribution, led to propose phenomenological parameterizations based on the generalized Navarro-Frank-White profile (GNFW) $[43,48]$. The functional form of the proposed profile is

$$
P_{e}(x)=1.65(h / 0.7)^{2} \mathrm{eVcm}^{-3} E^{8 / 3}(z) \times\left[\frac{M_{500}}{3 \times 10^{14}(0.7 / h) M_{\mathrm{e}}}\right]^{2 / 3+\alpha_{p}} p(x)
$$

where $\alpha_{p}=0.12, \alpha_{p}=0.12, E(z)=\left[\grave{U}_{m}(1+z)^{3}+\grave{U}_{\grave{E}}\right]^{1 / 2}$ for $\Lambda \mathrm{CDM}$ and

$$
p(x) \equiv \frac{P_{0}}{\left(c_{500} x\right)^{\gamma}\left[1+\left(c_{500} x\right)^{\alpha}\right]^{(\beta-\gamma) / \alpha}}
$$

The parameters were firstly derived using 33 X-Ray selected massive clusters $\left(M_{500}=\left[\begin{array}{ll}10^{14} & -10^{15}\end{array}\right]\right)$ up to redshift $\mathrm{Z} \leq 0.2$. Their best fit values were the following ones: $\left[c_{500}, \alpha, \beta, \gamma, P_{0}\right]=\left(1.177,1.051,5.4905,0.3081,8.403 h_{70}^{3 / 2}\right)$. Recently, combining TSZ and X-Ray data, the Planck Collaboration had fit the pressure profiles out to $3 r_{500}$ for a sample of 62 nearby massive clusters obtaining: $\quad\left[C_{500}, \alpha, \beta, \gamma, \mathrm{P}_{0}\right]=(1.81,1.33,4.13,0.31,6.41) \quad[49-$ 51]. Similarly, a study carried on 45 massive clusters with median mass $M_{500}=9 \times 10^{14} M_{\mathrm{e}}$ and median redshift $\mathrm{Z}=0.42$ found $\left[C_{500}, \alpha, \beta, \gamma\right.$ $\left.\mathrm{P}_{0}\right]=(1.18,0.86,3.67,0.67,4.29)[52]$.

TSZ surveys, such as the ones carried out by the Atacama Cosmology Telescope (ACT, [53]) the South Pole Telescope (SPT, [54]) and the Planck satellite [55], will provide new observational dataset, and they will offer new possibilities of testing modified gravity models on Mpc scales by fitting the distribution of the ICM within the modified gravitational potential well.

\section{Thermal Sunyaev-Zeldovich effect}

Cosmic Microwave Background (CMB) photons cross galaxy clusters, and they are scattered off by the free electrons of the ICM via an inverse Compton scattering. Thus, galaxy clusters distort the CMB blackbody spectrum, and produce secondary temperature anisotropies that are imprinted on the $\mathrm{CMB}$ radiation. Two components can distinguished: the thermal component (TSZ, [34]) due to the thermal motion of the electrons in the potential well of the cluster, and the kinematic (KSZ, [56]) component due to the proper motion of the galaxy cluster as a whole with respect to the CMB rest frame. Neglecting relativistic corrections in the electron temperature $\left(\mathrm{T}_{\mathrm{e}}\right)$, the TSZ and KSZ effects are given by

$$
\frac{\Delta T_{T S Z}(\hat{n})}{T_{0}}=\int \tilde{z}\left[G(\tilde{v}) \frac{k_{B} T_{e}}{m_{e} c^{2}}+\frac{\vec{v}_{c l}}{c}\right] \hat{n} d \tau,
$$

where, $\hat{n}$ is the direction of the cluster, $K_{\mathrm{B}}$ is the Boltzmann constant, $m_{\mathrm{e}} \mathrm{c}^{2}$ is the electron annihilation temperature, $\mathrm{c}$ is the speed of light, $\tilde{v}=h v / k_{B} T_{e}$ is the reduced frequency of observation, and $\vec{v}_{c l}$ is the peculiar velocity of the cluster. We denoted $d \tau=\sigma_{T} n_{e} d l$ the cluster optical depth to the SZ effect, with $\sigma_{\mathrm{T}}$ Thomson cross section, $n_{e}(l)$ the electron density evaluated along the line of sight $l ; \mathrm{T}_{0}$ is the current CMB blackbody temperature andG $(\tilde{v})$ is the frequency dependence of TSZ effect that, in the non relativistic limit $\left(\mathrm{T}_{\mathrm{e}} \approx \mathrm{few} \mathrm{keV}\right)$, is given by: $G(v)=\tilde{v} \operatorname{coth}(\tilde{v} / 2)-4$. For very hot clusters $\left(\mathrm{T}_{\mathrm{e}}>10 \mathrm{keV}\right)$, relativistic corrections must be included [57-59].

\section{Methodology}

The TSZ temperature anisotropies are usually expressed as the integral of the pressure profile along the line of sight:

$$
\frac{\Delta T_{T S Z}(\hat{n})}{T_{0}}=G(v) \frac{\sigma_{\mathrm{T}}}{m_{e} c^{2}} \int_{l} P_{\mathrm{e}}(l) d l
$$

To test modified theories of gravity using TSZ effect, the pressure profile $\left(\mathrm{p}_{\mathrm{e}}\right)$ must be specified. It can be computed integrating the following system of differential equations

$$
\begin{aligned}
& \frac{d \mathbb{P}(r)}{d r}=-\rho_{\text {gas }}(r) \frac{d \Phi_{\mathrm{eff}}(r)}{d r} \\
& \mathbb{P}(r) \propto \rho_{\text {gas }}^{\gamma}(r) \\
& \frac{d M(r)}{d r}=4 \pi \rho_{\text {gas }}(r) \\
& P_{e}(r)=P_{c} \mathbb{P}(r)
\end{aligned}
$$

where in the case of $f(\mathrm{R})$-gravity, the effective gravitational potential takes the following functional form $[15,16]$

$$
\Phi_{\mathrm{e} f f}(r)=\frac{\Phi_{\mathrm{N}}(r)}{1+\delta}-\frac{G \delta}{1+\delta} \int \frac{\rho\left(r^{\prime}\right) e^{-\frac{\left|r-r^{\prime}\right|}{L}}}{\left|r-r^{\prime}\right|} d^{3} r^{\prime}
$$


while, for MOG gravity model, it becomes

$$
\Phi_{\mathrm{e} f f}(r)=\Phi_{\mathrm{N}}(\mathrm{r})(\boldsymbol{z}+\alpha)+G \alpha \int \frac{\rho_{r}\left(r^{\prime}\right) e^{-\frac{\left|r-r^{\prime}\right|}{\mu^{*}}}}{\left|r-r^{\prime}\right|} d^{3} r
$$

In both cases $\Phi_{\mathrm{N}}(r)=-\frac{G M(r)}{r}$ is the classical Newtonian potential. Let us remark that the model does not include any DM component, but it assumes that baryons follow the modified gravitational potential well described in Equations (9) and (10) for $f(R)$-gravity and MOG models, respectively. When integrating the system of equations (5)-(8), one is assuming that: (i) the gas is in hydrostatic equilibrium within the modified potential well; (ii) the gas distribution is spherically symmetric; and (iii) the state of gas can be described with a polytropic equation of state (equation (6)). Under these assumptions, the system of equations (5)-(8), plus the equation for the modified gravitational potential, constitutes a closed system that can be integrated numerically to compute the pressure profile in ETGs, and to use TSZ anisotropies to constraint the theoretical parameters.

\section{Foreground cleaned Planck 2013 nominal data: Coma cluster}

Planck 2013 Nominal maps were used to measure the TSZ cluster profile and constrain the parameters of the modified gravitational potential of both theories. The publicly available Planck Nominal maps contain the cosmological CMB signal, instrumental noise, TSZ and KSZ emissions, point and extended infrared sources, thermal dust and $\mathrm{CO}$ emissions, and other foreground contributions. Although TSZ has a unique frequency dependence, it is not possible to detect the TSZ anisotropies at cluster location in the Planck Nominal maps. The technique used to reduce such foregrounds emissions provides a cleaned patch of sky centered at the cluster position $x, P(v, x)$, of size $2^{\circ}$ $\times 2^{\circ}$. Before the cleaning procedure is applied, publicly available Planck CO maps must be subtracted to each frequency channels [60], then the highest frequency channel could be used as a template for the thermal dust emission [61]. Then, the cleaning procedure uses the highest frequency channel as a thermal dust template; at the frequency channel $v$ the cleaned patch is obtained as follows

$$
\mathcal{P}(v, x)=P(v, x)-w(v) P(857 \mathrm{GH} z, x)
$$

where the weight $w(v)$ are given by

$$
w(v)=\frac{\dot{\mathrm{O}}_{x \in \mathcal{R}} P(v, x) P(857 \mathrm{GHz}, x)}{\dot{\mathrm{O}}_{x \in \mathcal{R}}[P(857 G H z, x)]^{2}}
$$

with $\mathcal{R}=\left[\theta_{c l}, \theta_{\text {patch }}\right]$ denoting the area where the weights are computed. Since the instruments operating at the frequencies (30-70 $\mathrm{GHz}$ ) have angular resolution higher than 10 arcminutes, only High Frequency Instrument (HFI) data at frequencies from 100 to $857 \mathrm{GHz}$ were considered.

As first attempt to build a new test, the study was particularized to the Coma cluster since is located close to the galactic pole where the foreground emission is comparatively low. The TSZ cluster profile was measured at 100,143 , and $353 \mathrm{GHz}$ channels, since the data at $545 \mathrm{GHz}$ were still dominated by the residuals of the dust emission. In Figure 1, it is shown a patch around the Coma cluster before (first row) and after (second row) the cleaning procedure was applied to remove foregrounds. The patches clearly show that the Nominal data are dominated by the the intrinsic CMB temperature fluctuations and dust emission, while TSZ signal dominates in the cleaned ones.

Patches of sky centered at the position of A1656 (Coma cluster) at

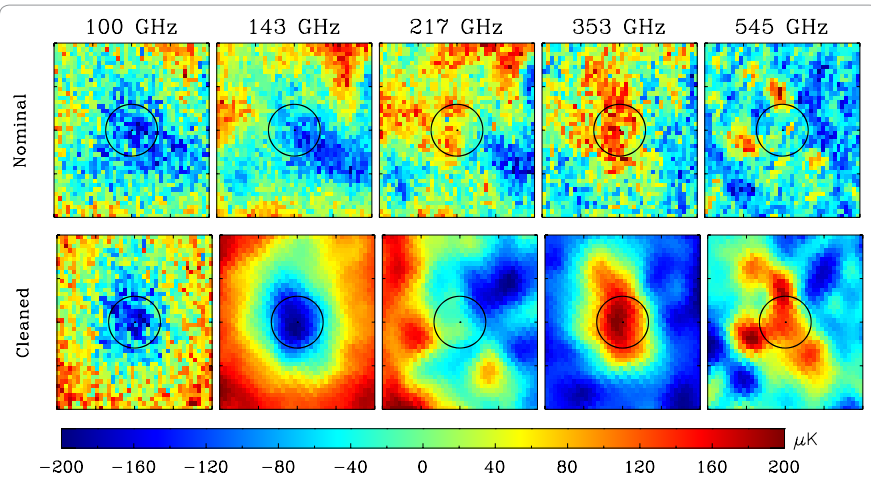

Figure 1: Patches of sky centered at the position of A1656 (Coma cluster) at $100-545 \mathrm{GHz}$. Patches have size of $2^{\circ} \times 2^{\circ}$. First row: Planck Nominal maps. Second row: foreground cleaned data.

\begin{tabular}{|c|c|c|c|}
\hline Theory & Parameter & Units & Priors \\
\hline \multirow{4}{*}{$4 * 90 f(R)$} & $\mathrm{p}_{\mathrm{c}}$ & $10^{-2} \mathrm{~cm}^{-3} \mathrm{keV}$ & {$[0.0,3.0]$} \\
\cline { 2 - 4 } & $\mathrm{Y}$ & - & $\frac{1.0,5}{3}$ \\
\cline { 2 - 4 } & $\mathrm{L}$ & $\mathrm{Mpc}$ & {$[0.1,20.0]$} \\
\cline { 2 - 4 } & $\delta$ & - & {$[-0.99,1.0]$} \\
\hline \multirow{3}{*}{$* 90 \mathrm{MOG}$} & $\mathrm{P}_{\mathrm{C}}$ & $10^{-2} \mathrm{~cm}^{-3} \mathrm{keV}$ & {$[0.0,3.0]$} \\
\cline { 2 - 4 } & $\mathrm{Y}$ & - & $1.0,5$ \\
& $\mu^{*-1}$ & $\mathrm{Mpc}$ & {$[0.01,20.0]$} \\
\hline & $\alpha$ & - & {$[0.1,20.0]$} \\
\hline
\end{tabular}

Table 1: MCMC priors of the explored parameter space.

100-545 GHz. Patches have size of $2^{\circ} \times 2^{\circ}$ First row: Planck Nominal maps. Second row: foreground cleaned data.

\section{MCMC priors}

The model parameters were constrained using a Monte Carlo Markov chain (MCMC) technique. The pipeline employed a Metropolis-Hastings sampling algorithm and used the Gelman-Rubin criteria to test the mixing and convergence of the runs. For each model, four independent chains have been run and the starting points of the parameter space have been randomly chosen. Each run contains at least 40,000 steps with an adapted step size to reach an optimal acceptance rate and to avoid poor mixing and slow convergence [62- 66]. The parameter space explored for both $f(\mathrm{R})$-gravity and MOG models is given in Table 1.

The TSZ data have been measured averaging the emission over disc/rings out to $\sim 100$ arcminutes from center of the Coma cluster. The galaxy cluster was assumed to be spherically symmetric and the ICM to be in hydrostatic equilibrium. The model predictions have been computed at the same apertures to compute the likelihood $-2 \log L=\chi^{2}\left(p, v_{k}\right)$ :

$$
-2 \log L=\chi^{2}\left(p, v_{k}\right)=\sum_{i, j=0}^{N} \Delta \bar{T}_{k i}(p) C_{i j}^{-1} \Delta \bar{T}_{k j}(p)
$$

In the previous equation, $\ddot{\mathrm{A}} \bar{T}_{k i}(p) \equiv \frac{\delta \bar{T}\left(p, v_{k}, \theta_{i}\right)}{T_{0}}-\frac{\delta \bar{T}\left(v_{k}, \theta_{i}\right)}{T_{0}}$ , where $\delta \bar{T}\left(p, v_{k}, \theta_{i}\right) / T_{0}$ are the data and $\delta \bar{T}\left(p, v_{k}, \theta_{i}\right) / T_{0}$ are the theoretical counterpart. Then, $N=22$ is the number of data points, $p$ are the parameters of the model, $C_{i j}$ denotes each Planck channel and $C_{i j}$ is the correlation matrix $[24,25,30]$. 


\begin{tabular}{|c|c|c|c|}
\hline Theory & Parameter & Units & Results \\
\hline \multirow{4}{*}{$* 90 f(R)$} & $\mathrm{P}_{\mathrm{c}}$ & $10^{-2} \mathrm{~cm}^{-3} \mathrm{keV}$ & $0.90 \pm 0.04$ \\
\cline { 2 - 4 } & $\mathrm{V}$ & - & $1.44_{-0.17}^{+0.10}$ \\
\cline { 2 - 4 } & $\mathrm{L}$ & $\mathrm{Mpc}$ & $2.19 \pm 1.02$ \\
\cline { 2 - 4 } & $\delta$ & - & $-0.48 \pm 0.22$ \\
\hline \multirow{3}{*}{ *90MOG } & $\mathrm{P}_{\mathrm{c}}$ & $10^{-2} \mathrm{~cm}^{-3} \mathrm{keV}$ & $0.77 \pm 0.03$ \\
\cline { 2 - 4 } & $\mathrm{V}$ & - & $1.40_{-0.13}^{+0.15}$ \\
\cline { 2 - 4 } & $\mu^{*-1}$ & $\mathrm{Mpc}$ & $4.22_{-1.08}^{+0.55}$ \\
\cline { 2 - 4 } & $\alpha$ & - & $6.68_{-2.08}^{+3.36}$ \\
\hline
\end{tabular}

Table 2: Best fit parameters for both $f(R)$-gravity and MOG.
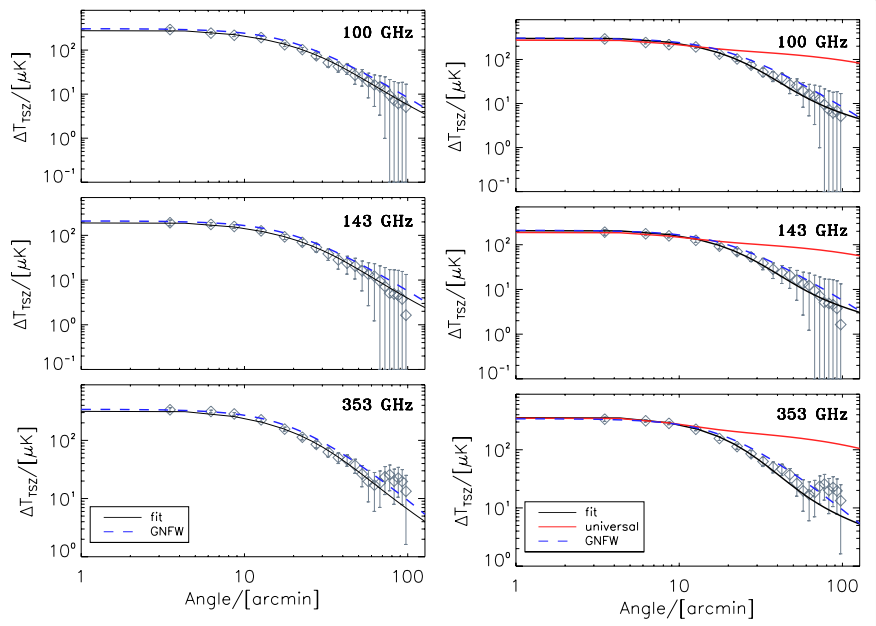

Figure 2: Best fitting model of $f(R)$-gravity and MOG in panels left and right, respectively. The black lines represent the best-fit models, the blue lines show the GNFW profile with best fit parameter from [43], and the red line in the right panel illustrates the MOG model with parameter fixed to their "universal" values [75]

\section{Results and Discussion}

MCMC were run to fit separately the data at each frequency channel. Then, the joint likelihood has been computed. The best fit parameters are summarized in Table 2, and the best fit models are shown in Figure $2[24,25,30]$.

\section{$f(R)$-gravity MOG}

Best fitting model of $f(R)$-gravity and MOG in panels left and right, respectively. The black lines represent the best-fit models, the blue lines show the GNFW profile with best fit parameter from [43], and the red line in the right panel illustrates the MOG model with parameter fixed to their "universal" values [67].

The analysis gave rise to some interesting results that can be summarized as follows:

- $f(R)$-theory: the strength of the modified potential in eq. (9) is $\delta \neq 0$ at the $95 \%$ of confidence level (CL). Therefore, the data are compatible with $f(R)$-gravity plus baryons. Next, the scale length of the potential $L$ is not equal to zero at the $95 \%$ CL. This limit corresponds to a Newtonian gravitational potential generated by an effective mass $M^{\prime}=M /(1+\delta)$. Since the data favors models with $\delta<0, M \square M$ would be analogue to the field generated by a cluster containing a large fraction of DM distributed like the baryonic gas [24,25].

- MOG theory: the strength of the Yukawa potential in eq. (10), $\alpha$, is compatible at $68 \% \mathrm{CL}$ with its universal value $\alpha \cong 8.89[14,67]$.
Whereas the universal value of scale length $\mu^{*-1}$ is ruled out at more than 3.5 $\sigma$. Therefore, the assumption that the parameters of the Yukawapotential can be assumed scale independent is also ruled out [30]. In fact, with this assumption, MOG is capable to fit only the central region ( $\theta 15$ arcminutes) of the galaxy cluster, while it overestimates the TSZ emission at larger apertures: at $\theta \sim 1$ degree the departure from the data is almost one order of magnitude (red dashed line in Figure 2).

For both theories, $f(\mathrm{R})$ and MOG, the polytropic index is consistent at $\sim 1.5 \sigma$ level with the value $\gamma \sim 1.2$ preferred by observations and numerical simulations within the $\Lambda \mathrm{CDM}$ concordance model. Since the physical state of the gas in a galaxy cluster is determined by its formation and evolution [68], the results could be interpreted as an indication that both $f(\mathrm{R})$ and MOG could be able to explain the emergence of the large scale structure as well as the concordance model.

\section{Conclusion}

Several theories of gravity have been constrained using cluster of galaxies such as chameleon $f(\mathrm{R})$ models [69-71], Galileon model [72], and K-mouflage gravity [73]. Here, we have reviewed the analysis that has been done to constrain those theories that modify the Newtonian potential in their weak filed limit by adding a Yukawa-like term. Specifically, we have reviewed the analysis on analytical $f(\mathrm{R})$-gravity and MOG models presented in [24,25,30]. Such a particular class of ETGs have been constrained using the TSZ temperature anisotropies due to the Coma cluster. With respect to other works, the model assumes that: (a) the hot ICM is in hydrostatic equilibrium within the modified gravitational potential wells described by the Equations (9) and (10) for $f(\mathrm{R})$-gravity and MOG, respectively; (b) the physical state of the gas is well described by a polytropic equation of state. These models have been tested using a foreground cleaned version of the 2013 data release of Planck Nominal maps. The measured TSZ profile at the location of Coma cluster has been used to constrain the model parameters employing a MCMC algorithm. Both analyses, for $f(\mathrm{R})$ and MOG, show the capability of the two theories to describe galaxy cluster without accounting for a DM component. However, the analyses are based on the assumptions of hydrostatic equilibrium and spherical symmetry of the cluster. Although such assumptions hold in the intermediate region of the Coma cluster where models are tested, they do not hold in general due to the presence of substructures, turbulences, and physical processes that can heat up or cool down the gas in the cluster core [74-81]. The departure from the spherical symmetry could affect both the innermost and outermost regions of Coma cluster. This is figured out in the the degeneracy between the strength of the gravitational potential, $\delta$ and $\alpha$, and the polytropic index of the gas $[25,30]$. Neverthless, a deeper study of this degeneracy should use N-body hydrodynamical simulations carried out for each set of parameter. Another limitation of the analysis was the angular resolution of the foreground cleaned data ( $F W H M=10$ arcminutes). This could be overcame with the next-generation of full sky CMB missions such as COrE/PRISM [82] that will have a much higher angular resolution and frequency coverage providing a powerful tool to properly investigate the relation between the underlying theory of gravity and the baryonic processes.

\section{References}

1. Riess AG, Strolger LG, Tonry J, Casertano S, Ferguson HC et al.(2004) Type la Supernova Discoveries at $Z>1$ from the Hubble Space Telescope: Evidence for Past Deceleration and Constraints on Dark Energy Evolution. Astrophys. J. 607: 655 .

2. Astier P, Guy J, Regnault N, Pain R, Aubourg E et al. (2006) The Supernova Legacy Survey: measurement of $\Omega_{\mathrm{M}}, \Omega_{\wedge}$ and $w$ from the first year data set. Astron. Astrophys. 447: 3. 
Citation: De Martino I (2017) Constraining Modified Gravity Models Using the Thermal Sunyaev-Zeldovich Effect. J Astrophys Aerospace Technol 5: 150. doi:10.4172/2329-6542.1000150

Page 5 of 6

3. Clocchiatti A, Schmidt B P, Filippenko A V, Challis P, Coil A L, et al., (2006) Hubble Space Telescope and Ground-based Observations of Type la Supernovae at Redshift 0.5: Cosmological Implications. Astrophys. J. 642: 1.

4. Blake C, Kazin EA, Beutler F, Davis TM, Parkinson D et al., (2011) The WiggleZ Dark Energy Survey: mapping the distance-redshift relation with baryon acoustic oscillations. Mon. Not. R. Astron. Soc. 418: 1707

5. Hinshaw G, Larson D, Komatsu E, Spergel D N, Bennett C L, et al., (2013) Nine-year Wilkinson Microwave Anisotropy Probe (WMAP) Observations: Cosmological Parameter Results. Astrophys. J. Suppl. 208: 19H.

6. Ade PAR, Aghanim N, Arnaud M, Ashdown M, Aumont $\mathrm{J}$ et al. Planck Collaboration (2015) Planck 2015 results. XIII. Cosmological parameters. Astron. Astrophys. 594: A13.

7. Quandt I, Schmidt HJ (1991) The Newtonian Limit of Fourth and Higher Order Gravity. Astron. Nachr. 312: 97.

8. Carroll SM, Duvvuri V, Trodden M, Turner MS (2004) Is cosmic speed-up due to new gravitational physics? Phys Rev D 70: 043528.

9. Sotiriou TP, Faroni V (2010) $f(R)$ theories of gravity. Rev Mod Phys, 82: 451

10. Nojiri S, Odintsov SD (2006) Modified $f(R)$ gravity consistent with realistic cosmology: From a matter dominated epoch to a dark energy universe. Phys. Rev. D 74: 086005.

11. Nojiri S, Odintsov SD (2011) Unified cosmic history in modified gravity: From $F(R)$ theory to Lorentz non-invariant models. Phys. Rept. 505: 59.

12. Capozziello S, De Laurentis M (2011) Extended Theories of Gravity. Phys Rept. 509: 167

13. Moffat JW (2006) Scalar tensor vector gravity theory J. Cosmol. Astropart Phys. 0603004

14. Moffat JW, Rahvar S (2013) The MOG weak field approximation and observational test of galaxy rotation curves. Mon. Not. R. Astron. Soc. 436 1439-1451.

15. Capozziello S, Stabile A, Troisi A (2012) Fourth-Order Gravity and Experimental Constraints on Eddington Parameters. Modern Physics Letters A 24: 09.

16. Capozziello $S$, De Laurentis $M(2012)$ The dark matter problem from $f(R)$ gravity viewpoint. Ann. Phys. 524:1.

17. Capozziello S, De Laurentis M, Odintsov SD, Stabile A (2011) Hydrostatic equilibrium and stellar structure in $f(R)$ gravity. Phys. Rev. D 83, 064004.

18. Capozziello S, De Laurentis M, De Martino I, Formisano M, Odintsov SD (2012) Jeans analysis of self-gravitating systems in $f(R)$ gravity. Phys Rev $D, 85$, 044022.

19. De Laurentis M, De Martino I (2013) Testing $f(R)$ theories using the first time derivative of the orbital period of the binary pulsars. Mon. Not. R. Astron. Soc. 43: 741

20. De Laurentis M, De Martino I (2015) Probing the physical and mathematica structure of $f(R)$-gravity by PSR $\mathrm{J} 0348+0432$. Int. J. Geom. Methods Mod. Phys. 12: 1550040 .

21. Cardone VF, Capozziello S (2011) Systematic biases on galaxy haloes parameters from Yukawa-like gravitational potentials. Mon. Not. R. Astron. Soc. 414: 1301.

22. Napolitano NR, Capozziello S, Romanowsky AJ, Capaccioli M, Tortora C (2012) Testing Yukawa-like Potentials from $f(R)$-gravity in Elliptical Galaxies. Astrophys. J. 748: 87.

23. Capozziello S, De Filippis E, Salzano V (2009) Modelling clusters of galaxies by $f(R)$ gravity. Mon. Not. R. Astron. Soc. 394: 947

24. De Martino I, De Laurentis M, Atrio-Barandela F, Capozziello S (2014) Constraining $f(R)$ gravity with Planck data on galaxy cluster profiles. Mon. Not. R. Astron. Soc. 442: 921.

25. De Martino I (2016) $f(R)$-gravity model of the Sunyaev-Zeldovich profile of the Coma cluster compatible with Planck data Phys. Rev. D 93: 4043.

26. De Martino I, De Laurentis M, Capozziello S (2015) Constraining $f(R)$ gravity by the Large Scale Structure. Universe 1: 123.

27. Brownstein JR, Moffat JW (2006) Galaxy cluster masses without non-baryonic dark matter. Mon. Not. R. Astron. Soc. 367: 527
28. Brownstein JR, Moffat JW (2006) Galaxy Rotation Curves without Nonbaryonic Dark Matter. Astrophys. J. 636: 721.

29. Brownstein J R, Moffat JW (2007) The Bullet Cluster 1E0657-558 evidence shows modified gravity in the absence of dark matter. Mon. Not. R. Astron. Soc. 382: 29.

30. De Martino I, de Laurentis M (2017) On the universality of MOG weak field approximation at galaxy cluster scale. Phys. Lett. B 770: 440

31. Moffat JW (2006) Late-time inhomogeneity and acceleration without dark energy J. Cosmol. Astropart. Phys. 05: 0010

32. Moffat JW (2007) A modified gravity and its consequences for the solar system astrophysics and cosmology int. J. Mod. Phys. 16 (2007) 2075

33. Moffat JW, Toth VT (2009) The bending of light and lensing in modified gravity Mon. Not. R. Astron. Soc. 397: 1885.

34. Sunyaev RA, Zeldovich YB (1972) The Observations of Relic Radiation as a Test of the Nature of X-Ray Radiation from the Clusters of Galaxies. Comments on Astrophys. Space Phys. 4: 173.

35. White SDM, Navarro JF, Evrard AE, Frenk CS (1993) Nature 366: 429-433.

36. White D A, Fabian A C (1995) Einstein Observatory evidence for the widespread baryon overdensity in clusters of galaxies Mon. Not. R. Astron. Soc. 273: 72-84.

37. Lubin LM, Cen R, Bahcall NA, Ostriker JP (1996) The Baryon Fraction and Velocity--Temperature Relation in Galaxy Clusters : Models versus Observations Astrophys. J. 460: 10

38. Kaiser N (1986) Evolution and clustering of rich clusters. Mon. Not. R. Astron Soc. 222: 323.

39. Kravtsov AV, Borgani S (2012) Formation of Galaxy Clusters. Annual Review of Astronomy and Astrophysics, 50, 353-409.

40. Böhringer H, Schuecker P, Pratt G, Arnaud M, Ponman T, et al. (2007). The representative XMM-Newton cluster structure survey (REXCESS) of an X-ray luminosity selected galaxy cluster sample. A\&A, 469: 363-377.

41. Bonamente M, Joy M, La Roque S J, Carlstrom J E, Nagai D et al. (2008) Scaling Relations from Sunyaev-Zel'dovich Effect and Chandra X-Ray Measurements of High-Redshift Galaxy Clusters. Astrophys. J 675: 106.

42. Marrone D P Smith G P, Richard J, Marshall J, Bonamente M, et al. (2009) LoCuSS: A Comparison of Sunyaev-Zel'dovich Effect and GravitationalLensing Measurements of Galaxy Clusters. Astrophys. J 701: L114.

43. Arnaud M, Pratt G W, Piffaretti R, Böhringer H, Croston J H et al. (2010) The universal galaxy cluster pressure profile from a representative sample of nearby systems (REXCESS) and the $\mathrm{Y}_{\mathrm{sz}}-\mathrm{M}_{500}$ relation. Astron. Astrophys. 517: 92.

44. Melin, J., Bartlett, J. G., Delabrouille, J., Arnaud M, Piffaretti R et al. (2011) The galaxy cluster YSZ - LX and YSZ - M relations from the WMAP 5-yr data Astron. Astrophys. 525: A139.

45. Andersson K, Benson BA, Ade PAR, Aird KA, Armstrong B et al. (2011) X-Ray Properties of the First Sunyaev-Zel'dovich Effect Selected Galaxy Cluster Sample from the South Pole Telescope. Astrophys. J. 738: 48.

46. De Martino I, Atrio-Barandela F (2016) SZ/X-ray scaling relations using X-ray data and Planck Nominal maps. Mon. Not. R. Astron. Soc. 461, 3: 3222-3232.

47. Jimeno P, Diego JM, Broadhurst T, De Martino I, Lazkoz R (2017) Planck/SDSS Cluster Mass and Gas Scaling Relations for a Volume-Complete redMaPPer Sample. arXiv:1706.00395

48. Navarro J F, Frenk CS, White SDM (1997), A Universal Density Profile from Hierarchical Clustering. Astrophys. J. 490: 493.

49. Planck Collaboration, 2011a, "Planck Early Results X: Statistical analysis of Sunyaev-Zeldovich scaling relations for X-ray galaxy clusters", A\&A, 536, A10.

50. Ade P A R, Aghanim N, Arnaud M, Ashdown M, Aumont J, et al. (2011) Planck Early Results. XI. Calibration of the local galaxy cluster Sunyaev-Zeldovich scaling relations. Astron. Astrophys. 536: A11.

51. Ade P A R, Aghanim N, Arnaud M, Ashdown M, Atrio-Barandela F et al. (2013) Planck Intermediate Results $\mathrm{V}$ : Pressure profiles of galaxy clusters from the Planck Survey. Astron. Astrophys. 550: 131

52. Sayers J, Czakon NG, Mantz A, Golwala S R, Ameglio S, et al. (2013) Sunyaev-Zel'dovich-measured Pressure Profiles from the Bolocam X-Ray/SZ Galaxy Cluster Sample. Astrophys. J. 768: 177 
Citation: De Martino I (2017) Constraining Modified Gravity Models Using the Thermal Sunyaev-Zeldovich Effect. J Astrophys Aerospace Technol 5: 150. doi:10.4172/2329-6542.1000150

Page 6 of 6

53. Kosowsky A (2003) The Atacama Cosmology Telescope. New Astron. Rev. 47: 939.

54. Carlstrom JE, Ade PAR, Aird KA, Benson BA, Bleem LE et al. (2011) The 10 Meter South Pole Telescope Publ. Astron. Soc. Pac 123: 56.

55. Ade PAR, Aghanim N, Alves MIR, Armitage-Caplan C, Arnaud M et al. (2014) Planck 2013 results. I.Overview of products and scientific results. Astron. Astrophys. 571: A1.

56. Sunyaev R, Zeldovich $Y(1980)$ The velocity of clusters of galaxies relative to the microwave background - The possibility of its measurement. Mon. Not. R. Astron. Soc. 190:413

57. Itoh N, Kohyama Y, Nozawa S, (1998) Relativistic Corrections to the SunyaevZeldovich Effect for Clusters of Galaxies. Astrophys. J. 5027.

58. Nozawa S, Itoh N, Kohyama Y, (1998) Relativistic Corrections to the SunyaevZeldovich Effect for Clusters of Galaxies. II. Inclusion of Peculiar Velocities. Astrophys. J. 508: 17.

59. Nozawa S, Itoh N, Suda $Y$, Ohhata $Y$, (2006) An improved formula for the relativistic corrections to the kinematical Sunyaev-Zeldovich effect for clusters of galaxies. Nuovo Cimento 121: 487.

60. Ade P A R, Aghanim N, Alves M I R, Armitage-Caplan C, Arnaud M et al. (2014) Planck 2013 results. XIII. Galactic CO emission. Astron. Astrophys. 571: 13.

61. De Martino I, Génova-Santos R, Atrio-Barandela F, Ebeling H, Kashlinsky A, et al. (2015) Constraining the Redshift Evolution of the Cosmic Microwave Background Blackbody Temperature with PLANCK Data. Astrophys. J. 808: 128.

62. Hastings W K (1970) Monte Carlo sampling methods using Markov chains and their applications. Biometrika 57: 97-109.

63. Metropolis N, Rosenbluth A W, Rosenbluth M N, Teller A H (1953) Equation of State Calculations by Fast Computing Machines. J. Chem., Phys. 21: 1087.

64. Gelman A, Rubin D B, (1992) Inference from Iterative Simulation Using Multiple Sequences. Statist. Sci. 7: 457

65. Gelman A, Roberts G O, Gilks W R, (1996) Efficient Metropolis jumping rules. Bayesian statistics 5: 599-608.

66. Roberts G O, Gelman A, Gilks W R, (1997) Weak convergence and optimal scaling of random walk Metropolis algorithms. Ann. Appl. Probab. 7, 1: 110.

67. Moffat J W, Rahvar S (2014) The MOG weak field approximation - II Observational test of Chandra X-ray clusters. Mon. Not. R. Astron. Soc. 441: 3724

68. Bertschinger E (1985) Self-similar secondary infall and accretion in an Einstein- de Sitter universe. Astrophys. J. Suppl. 58: 39.

69. Terukina A, Lombriser L, Yamamoto K, Bacon D, Koyama K et al. (2014) Testing chameleon gravity with the Coma cluster. J. Cosmol. Astropart. Phys. 04: 013.

70. Wilcox, H, Bacon D, Nichol R C, Rooney P J, Terukina A et al. (2015) The XMM Cluster Survey: testing chameleon gravity using the profiles of clusters. Mon. Not. R. Astron. Soc. 452: 1171.

71. Li B, He J H, Gao L (2015) Cluster gas fraction as a test of gravity. Mon. Not R. Astron. Soc. 456: 146.

72. Terukina A, Yamamoto K, Okabe N, Matsushita K, Sasaki T. (2015) Testing a generalized cubic Galileon gravity model with the Coma Cluster. J. Cosmol. Astropart. Phys. 10: 64

73. Brax P, Rizzo LA, Valageas $P$ (2015) K-mouflage effects on clusters of galaxies Phys. Rev. D 92: 043519

74. Arlen T, Aune T, Beilicke M, Benbow W,Bouvier A. et al. (2012) Constraints on Cosmic Rays, Magnetic Fields, and Dark Matter from Gamma-Ray Observations of the Coma Cluster of Galaxies with VERITAS and Fermi. Astrophys. J. 757: 123

75. Churazov E, Vikhlinin A, Zhuravleva I, Schekochihin A, Parrish I, et al. (2012) $X$-ray surface brightness and gas density fluctuations in the Coma cluster. Mon. Not. R. Astron. Soc. 421: 1123.

76. Simionescu A, Werner N, Urban O, Allen SW, Fabian AC et al. (2013) Thermodynamics of the Coma Cluster Outskirts. Astrophys. J. 775, 4.

77. Sato T, Matsushita K, Ota N, Sato K, Nakazawa K, Sarazin C L (2011) Suzaku Observations of Iron K-Lines from the Intracluster Medium of the Coma Cluster. Publ. Astron. Soc. Jpn. 63: 991

78. Neumann DM, Lumb DH, Pratt, GW ,Briel UG (2003) The dynamical state of the Coma cluster with XMM-Newton 400: 811-821.

79. Sanders J S, Fabian A C, Churazov E, Schekochihin A A, Simionescu A et al. (2013) Linear Structures in the Core of the Coma Cluster of Galaxies. Science 341: 6152.

80. Snowden S L, Mushotzky R F, Kuntz K D, Davis D S (2008) A catalog of galaxy clusters observed by XMM-Newton. Astron. Astrophys. 478: 615.

81. Wik D R, Sarazin C L, Finoguenov A, Matsushita K, Nakazawa K, et al. (2009) A Suzaku Search for Nonthermal Emission at Hard X-Ray Energies in the Coma Cluster. Astrophys. J. 696: 1700

82. André P, Baccigalupi C, Banday A, Barbosa D, Barreiro B et al. (2014) PRISM (Polarized Radiation Imaging and Spectroscopy Mission): an extended white paper. J. Cosmol. Astropart. Phys. 1402: 006. 